\title{
Desafios no Cuidado da Doença Renal Crônica nas Unidades de Atenção Primária à Saúde em Município Cearense
}

\author{
Challenges in Caring of Chronic Kidney Disease in Primary Health Care Units \\ in a city in the state of Ceará
}

\author{
Elayne Cristina Matias Nóbrega ${ }^{1}$ \\ Sâmela Matias Nóbrega² \\ Raquel Simões Monteiro Alves ${ }^{3}$ \\ Caroline Moreira Arruda ${ }^{3}$ \\ Sarah Maria Feitoza Souza ${ }^{4}$
}

\section{RESUMO}

Objetivo: O estudo analisou o cuidado à saúde de diabéticos e hipertensos quanto à prevenção, o diagnóstico e o tratamento de doença renal crônica (DRC) acompanhados em Unidades de Atenção Primária à Saúde (APS) no Município de Tauá, Ceará. Metodologia: Tratou-se de estudo de caso descritivo com abordagem qualitativa, no qual participaram 27 informantes, incluindo os profissionais de saúde de Unidades de APS; os pacientes com DRC em terapia renal substitutiva com diagnóstico de diabetes mellitus (DM) e/ou hipertensão arterial sistêmica (HAS) e os profissionais ligados à gestão da assistência à saúde local. Os dados foram obtidos pela realização de entrevistas individuais e analisados por análise temática. Resultados: Verificou-se lacunas na assistência aos pacientes diabéticos e hipertensos envolvendo tanto a APS quanto a atenção especializada, no que diz respeito à carência de medicamentos, exames e consultas especializadas; inexistência de contrarreferência e nefrologista para o acompanhamento dos casos. No âmbito da APS, observou-se um vínculo fragilizado e uma baixa adesão ao tratamento multiprofissional entre os pacientes com DM e/ou HAS, incluindo aqueles com DRC; as ações educativas não abordaram diretamente a prevenção de doenças renais e os profissionais de saúde necessitam de educação continuada sobre o manejo da DRC. Conclusão: O estudo revelou falhas no cuidado com os diabéticos e os hipertensos, sobressaindo-se o acesso insuficiente a medicamentos, exames e consultas especializadas; bem como o vínculo fragilizado e a baixa adesão destes pacientes ao tratamento multiprofissional no componente primário. Faz-se necessária a implantação de uma linha de cuidado para assistir aos usuários diabéticos e hipertensos com ou sem DRC no município em questão.

\section{DESCRITORES}

Insuficiência Renal Crônica. Diabetes Mellitus. Hipertensão. Atenção Primária à Saúde.

\begin{abstract}
Objective: The study analyzed the health care of diabetics and hypertensive patients regarding the prevention, diagnosis and treatment of CKD monitored in Primary Health Care (PHC) Units in the city of Tauá, Ceará. Methodology: It was a descriptive case study with a qualitative approach, in which 27 informants participated, including health professionals from PHC Units; CKD patients on Renal Replacement Therapy diagnosed with Diabetes Mellitus (DM) and/or Systemic Arterial Hypertension (SAH) and professionals connected to the management of local health care. The data were collected by conducting individual interviews and analyzed by thematic analysis. Results: Gaps in the assistance of diabetic and hypertensive patients involving both PHC and Specialized Care were found regarding the lack of medication, tests, and specialized consultations; lack of counter-referral and nephrologist to monitor cases. Within the PHC, a weak bond and low adherence to multiprofessional treatment were observed among patients with DM and / or $\mathrm{SAH}$, including those with CKD; educational actions do not directly address the prevention of kidney disease and health professionals need permanent education on the management of the DRC. Conclusion: The study revealed flaws in the care of diabetic and hypertensive patients, with insufficient access to medicines, exams and specialized consultations; as well as the weakened bond and low adherence of these patients to the multiprofessional treatment in the primary component. It is necessary to implement a line of care to assist diabetic and hypertensive users with or without RDC in the city in question.
\end{abstract}

\section{DESCRIPTORS}

Renal Insufficiency, Chronic. Diabetes Mellitus. Hypertension. Primary Health Care.

${ }^{1}$ Nutricionista. Residência Integrada em Saúde. Escola de Saúde Pública do Ceará - ESP/CE, Tauá, Ceará, Brasil.

${ }^{2}$ Psicóloga. Residência Integrada em Saúde. Escola de Saúde Pública do Ceará - ESP/CE, Tauá, Ceará, Brasil.

${ }^{3}$ Nutricionista. Mestre em Nutrição e Saúde. Universidade Estadual do Ceará - UECE, Fortaleza, Ceará, Brasil.

${ }^{4}$ Enfermeira. Mestre em Enfermagem. Universidade Federal do Ceará - UFC, Fortaleza, Ceará, Brasil. 
A doença renal crônica (DRC) é um dos principais problemas de saúde pública em todo o mundo. Trata-se de uma patologia de curso prolongado, com múltiplas causas e que, na maior parte do tempo de sua evolução, é assintomática. A DRC é classificada em cinco estágios, sendo os estágios iniciais tratados com terapia conservadora ou não dialítica a fim de controlar os fatores de risco para progressão da doença; enquanto o estágio 5 necessita de terapia renal substitutiva (TRS), uma modalidade de substituição da função renal, realizada por meio dos procedimentos de hemodiálise, diálise peritoneal e transplante renal. Ressalta-se que todos os estágios estão associados a riscos aumentados de morbidade cardiovascular, mortalidade prematura e/ou diminuição da qualidade de vida $^{1,2,3}$.

O Inquérito Brasileiro de Diálise Crônica mostrou que o número total estimado de pacientes no país em julho de 2017 foi de 126.583, indicando um aumento de $3 \% \mathrm{em}$ relação ao ano anterior. Destes, 58\% eram do sexo masculino, 93,1\% faziam tratamento por hemodiálise e 6,9\% por diálise peritoneal. As causas primárias da DRC mais frequentes em 2017 foram a hipertensão arterial (34\%) e o diabetes (31\%), sendo esta última o principal fator de entrada em diálise, embora não represente a maioria dos pacientes em tratamento, tendo em vista à maior mortalidade dos enfermos com esta patologia ${ }^{4}$.

$O$ excesso de glicose, presente nos pacientes com DM, é responsável por induzir uma inflamação sistêmica e intra-renal, desempenhando um papel crítico na patogênese da doença renal do diabetes, reconhecida atualmente como a principal causa de doença renal dialítica em todo o mundo. Os achados de uma revisão sistemática também apontaram a hipertensão arterial como precursora da DRC em $62,5 \%$ dos estudos analisados, com maior prevalência em indivíduos idosos ${ }^{5,6}$. Tais trabalhos corroboram a participação significativa dessas duas patologias na origem e no agravamento das doenças renais.

Diversos estudos apontam os impactos negativos que incidem sobre a vida de pessoas com DRC, especialmente entre os que realizam TRS. A maioria destes percebem-se como indivíduos disfuncionais e inúteis, principalmente devido a considerável dificuldade para permanecer e/ou retornar ao trabalho devido ao rigor exigido pelo tratamento, necessidade de adaptações e uma nova construção psíquica, contribuindo para o surgimento de sintomas depressivos, ansiedade, estresse e sensação de ser um incômodo para a família; além disso, consideram seu tratamento como privação de sua liberdade. Dessa forma, juntamente ao diagnóstico e tratamento, com suas consequentes mudanças fisiológicas e emocionais, diversas perdas acontecem nos âmbitos profissional, social, sexual e psicológico $^{7,8}$.

O sistema único de saúde (SUS) exerce um papel fundamental no atendimento ao paciente com DRC, sendo atualmente responsável pelo financiamento de $90 \%$ dos tratamentos de pacientes em TRS. Somente no ano de 2015, foram gastos mais de 2 bilhões com a TRS, correspondendo a mais de $5 \%$ dos gastos do SUS. Esses números representam um percentual importante dos gastos nacionais, gerando preocupações quanto à manutenção futura do tratamento da DRC com TRS no Brasil, sendo necessá- 
rio adotar políticas com medidas preventivas e tratamento em tempo oportuno, pois de acordo com os inquéritos populacionais, 3 a 6 milhões de brasileiros seriam renais crônicos; no entanto, pouco mais de 100 mil recebem terapia dialítica ${ }^{9,10}$.

Conforme determina a Portaria $\mathrm{n}^{0}$ 1.675, de 7 de junho de 2018, cabe à Atenção Primária à Saúde (APS) realizar ações de promoção da saúde, prevenção de doenças e agravos e controle das principais patologias relacionadas à $D R C$, como a hipertensão arterial sistêmica (HAS) e o diabetes mellitus (DM), considerados os fatores de risco mais prevalentes na população; realizar acolhimento com classificação e estratificação de risco e vulnerabilidade, diagnóstico precoce e tratamento oportuno da doença; coordenar o cuidado atuando como o centro de comunicação entre os diversos componentes da Rede de Atenção à Saúde (RAS) e ordenar os fluxos e contrafluxos de pessoas; realizar atividades educativas e apoiar o autocuidado, ampliando a autonomia da pessoa com DRC por meio de abordagem multiprofissional e intersetorial ${ }^{11}$.

Tendo em vista os diversos impactos socioeconômicos e na qualidade de vida dos pacientes com DRC, os custos elevados com o tratamento para o SUS e os riscos de mortalidade e comorbidades associadas, faz-se necessário acompanhar os pacientes incluídos nos grupos de risco para prevenir e/ ou diagnosticar precocemente a DRC, independente do estágio, no âmbito da Atenção Primária à Saúde. Portanto, o estudo teve como objetivo analisar o cuidado à saúde de diabéticos e hipertensos quanto à prevenção, diagnóstico e tratamento de DRC acompa- nhados em Unidades de Atenção Primária à Saúde no Município de Tauá, Ceará.

\section{METODOLOGIA}

Este trabalho consistiu em um estudo de caso descritivo com abordagem qualitativa, no qual foram incluídos três segmentos de informantes: profissionais da assistência à saúde, pacientes e profissionais da gestão. Participaram doze profissionais de saúde de seis Unidades de APS situadas na sede do Município de Tauá. Destes, quatro eram médicos, seis enfermeiros e dois nutricionistas. Foram incluídos 13 pacientes com DRC em TRS com diagnóstico de DM e/ou HAS, com idade média de 50 anos e período de tratamento de aproximadamente 56 meses. Participaram também dois profissionais ligados à gestão da assistência à saúde no âmbito do município.

A coleta de dados aconteceu nos meses de setembro e outubro de 2019, por meio de entrevistas individuais gravadas, após autorização do entrevistado, mediante assinatura do Termo de Consentimento Livre e Esclarecido. As entrevistas foram orientadas por roteiro semiestruturado formulado pelas próprias pesquisadoras. Foram excluídos do estudo os profissionais das Unidades localizadas nos distritos, em virtude da dificuldade de acesso a estas localidades e os pacientes com DRC sem diagnóstico de DM e/ou HAS como causa base da doença renal, sendo a amostra final quantificada pela técnica de saturação teórica, ou seja, as entrevistas foram realizadas até que não revelassem nenhuma informação nova ou relevante em relação ao objeto de estudo e as categorias bem esta- 
belecidas e validadas nos três segmentos de informantes entrevistados ${ }^{12}$.

Os dados foram analisados conforme pressupostos da Análise Temática referenciada por Minayo ${ }^{13}$, que a descreve como uma análise simples e considerada apropriada para investigações qualitativas em saúde. Baseado neste referencial, operacionalmente a análise do material foi organizada em três etapas: adotou-se como primeira operação a leitura compreensiva do material selecionado (entrevistas transcritas), no intuito de se ter uma visão de conjunto e de se apreender as particularidades do material.

Em seguida, partiu-se para a etapa de exploração do material, na qual foram destacados e analisados trechos emblemáticos das ideias explícitas e implícitas apreendidas pelos pesquisadores, resultando na elaboração de categorias temáticas. A última etapa consistiu na elaboração da síntese interpretativa com a qual se buscou encontrar elementos para embasar, interpretar e aprofundar as categorias levantadas e construir um relato articulado entre os objetivos do estudo e os sentidos mais amplos adjacentes às ideias emergidas das entrevistas. O corpus analisado por este estudo foi composto pela transcrição ipsis litteris das entrevistas realizadas com os informantes da pesquisa.

Para preservar a identidade dos informantes foram atribuídas às falas a letra "P" para profissionais da assistência à saúde e da gestão e "U" para usuários, acompanhados por número para diferenciar os indivíduos.

O trabalho foi aprovado pelo Comitê de Ética em Pesquisa da Escola de Saúde Pública do Ceará, sob o parecer $n^{\circ} 3.372 .458$, seguindo os preceitos éticos recomendados pela Resolução n. 466/2012, do Conselho Nacional de Saúde (CNS).

\section{RESULTADOS}

Após o processamento dos dados obtidos pela realização das entrevistas individuais, elencaram-se três categorias: "Fragilidades no cuidado compartilhado entre a atenção primária e a especializada", "(Des) vinculação do paciente na atenção primária à saúde" e "Falhas na abordagem preventiva no nível primário", descritas a seguir:

- Fragilidades no cuidado compartilhado entre a atenção primária e a especializada

A assistência aos pacientes diabéticos e hipertensos nas Unidades de APS no Município de Tauá acontece por meio de uma estratificação com classificação de risco, no qual os pacientes são acompanhados por equipe multiprofissional tanto da Atenção Primária quanto Especializada, em períodos determinados, de acordo com a classificação em baixo, médio, alto ou muito alto risco; no entanto, relatos evidenciaram uma descontinuidade no processo de estratificação por falta de exames e de consultas especializadas:

"Infelizmente no momento está parado, nós temos a estratificação de risco, e eles como alto risco e muito alto risco eram pra ser acompanhados na atenção secundária, na policlínica, só que está parado esse fluxo, então na Unidade a gente estratifica, mas eu não tenho pra onde encaminhar, eu só identifico que tenho, mas eu não tenho o que fazer" (P08) 
Uma das dificuldades vivenciadas pelos profissionais de saúde refere-se à inexistência de nefrologista para acompanhamento continuado de diabéticos e hipertensos estratificados na Atenção Primária, uma vez que o município não dispõe de serviço de nefrologia, sendo necessário o deslocamento dos pacientes para outra cidade, fato colocado como um risco para o surgimento de complicações e/ou comorbidades nestes pacientes:

"A primeira dificuldade seria a falta do especialista aqui no nosso município, não temos nefrologista atendendo na policlínica, então no caso da necessidade desse paciente ele tem que se deslocar para outra cidade, então a partir do momento que você diagnostica alguma alteração que precisa do especialista aí já tem essa dificuldade, pela necessidade de deslocamento, pela necessidade de ter que marcar, ter que esperar um pouco surgir vaga, então a falta do profissional aqui no município acaba comprometendo o diagnóstico precoce e o tratamento mais eficaz pra esse paciente" (P10)

A carência e/ou demora no acesso aos exames também é percebida pelos pacientes com DRC que realizam hemodiálise, evidenciando inconstância no cuidado longitudinal e entraves no funcionamento da RAS que perpassa tanto o componente primário quanto o secundário:

"Deveria ser mais rápido, o SUS demora muito os exames" (U03)
"Os exames consegue, mas não todos, ela marca mais na policlínica e assim mesmo tá difícil, tá é difícil ela marcar pra lá, ela tem um da tireóide faz é tempo que tá lá pra marcar não foi marcado, tem o do coração" (U12)

Verificou-se também falhas na comunicação entre a Atenção Primária e a Atenção Especializada relacionada à contrarreferência:

"A atenção primária nunca recebe a contrarreferência, pra sair daqui tem que sair com a referência, a classificação de risco, que é pra ele ser atendido em tempo hábil, mas quando ele vem de lá se ele for um paciente esclarecido e souber me falar como foi a consulta... ótimo, mas se não for...eu não sei o que aconteceu porque simplesmente eu não recebo uma contrarreferência, não existe" (P05)

- "(Des)vinculação do paciente na atenção primária à saúde"

Os profissionais relatam vínculo fragilizado dos pacientes diabéticos e hipertensos na Atenção Primária, existindo uma maior procura pelo médico para solicitação de renovação de receita de medicamentos:

"Nós temos muitos pacientes que não querem vir à unidade, muitos pacientes que a gente precisa ir a domicílio pra poder fazer esse acompanhamento, e muitos acham que precisam vir na Unidade só pra renovar receita, que não é o certo, então assim, junto aos 
agentes de saúde a gente tá tentando buscar esses pacientes, principalmente aqueles que não são estratificados ainda, pra vir a unidade, fazer essa estratificação, e a partir daí a gente iniciar esse processo de acompanhamento mais de perto" (P11)

"Os retornos se a gente não marcar eles não agendam de novo, que eles ainda tem aquela cultura de que só é pra vim se for pra pegar o remédio, senão não vem, só pra garantir pra olhar a pressão, é muito difícil, o diabético também é do mesmo jeito" (P05)

A baixa adesão ao tratamento multiprofissional também foi outro ponto elencado, uma realidade visualizada como um fator limitante para o controle das condições crônicas e a prevenção do aparecimento de comorbidades, incluindo as renais nos pacientes diabéticos e hipertensos:

"Sempre a maior dificuldade que tem é a adesão ao tratamento, os hipertensos até que é menos, porque os hipertensos normalmente sente uma dor de cabeça, alguma coisa, um dia incomoda e isso preocupa, mas os diabéticos como demora mais sentir alguma coisa é mais difícil ainda controlar, mesmo tendo toda a medicação possível, mas junto muito essa falta de adesão ao tratamento e no caso do diabético quando é um diabético mais avançado que necessita de insulina aí junta o preconceito com a insulina com medo de se furar, aí é mais dificuldade da adesão do que da falta do material humano e do medicamento" (P12)

Em relação aos pacientes renais crônicos também se percebe uma vinculação enfraquecida na Atenção Primária, sendo a procura restrita a busca por medicamentos, vacinas e encaminhamentos de exames:

"É difícil eu ir lá, só quando eu vou marcar um exame" (U03)

"É difícil, é difícil eu ir, eu vou ainda por causa das vacinas que a gente tem que tomar né, mas assim pra ir atrás do médico eu nunca mais fui não" (U05)

“Eu vou só pra pegar o remédio" (U06)

Muitos destes pacientes possuíam pouco ou nenhum vínculo com a unidade básica de saúde (UBS) antes do diagnóstico da DRC, mantendo-se nesta condição mesmo após o tratamento da doença renal. O agente comunitário de saúde (ACS) foi o profissional com maior contato com estes pacientes, auxiliando-os na marcação de consultas e/ou exames e distribuição de medicamentos:

"Antes deu adoecer eu nunca fui nem ao médico, eu nem sabia, porque a pessoa do interior, eu sou da roça né, aí a pessoa não liga muito, às vezes sentia algum problemazinha não ia ao médico nem nada, eu só fui mesmo no médico quando tava mesmo doente que não aguentei mais trabalhar" (U13)

“Não sei nem quem é o meu doutor 
de lá (referindo-se à UBS), o médico" (U09)

"Contato só com ela (agente de saúde), é tanto que ela mesma é quem, muitas vezes é ela quem resolve problema de medicação pra mim, ela é quem já me diz..."não esse aqui não tem, esse aqui não tem", é tanto que eu já nem vou no PSF minha medicação é toda comprada" (U05)

$\mathrm{Na}$ visão dos profissionais, os pacientes com DRC em TRS não se sentem parte da Unidade:

"Tenho um paciente renal crônico aqui na área, mas ele não vem pra Unidade porque ele já faz hemodiálise, então geralmente é solicitado a visita do médico ou do enfermeiro pra ver como é que ele tá, quem mais visita é a médica, mas aqui mesmo pra Unidade ele não chegou a vim, já por conta desse acompanhamento, que ainda tem esse problema, o fato dele já ser acompanhado em um serviço especializado, "não tem mais o que eu ir buscar no PSF", aí a gente fica sabendo mais notícias pelo agente de saúde do que mesmo pelo paciente" (P05)

Os pacientes em TRS justificam a falta de medicamentos e a baixa oferta de exames como a causa principal pela baixa frequência na UBS, revelando uma insuficiência no acesso a medicamentos e serviços essenciais para o manejo destes pacientes no município, além de comprometimento na resolutividade e atendimento integral na rede de atenção local:
“Eu vou lá (UBS) só quando tem que buscar algum remédio, mas eu quase não frequento não sabe porque... porque vai quando chega lá não acha remédio, a gente leva a receita daqui quando chega lá, as vezes tem uns mas não tem tudo, eu me arrisco até comprar" (U08)

Outros pacientes acreditam que o tratamento no serviço especializado em nefrologia, realizado em município vizinho, é suficiente para atender suas necessidades de saúde e/ou julgam estar em boa condição clínica não havendo necessidade de ser acompanhado na unidade no momento:

"Porque assim eu graças a Deus tô bem né, aí eu venho pra cá $3 x$ por semana (centro de nefrologia), aí não precisa eu ir pro PSF" (U13)

"Não...aqui eu já tenho tudo que eu preciso (referindo-se ao centro de nefrologia)" (U05)

- "Falhas na abordagem preventiva no nível primário"

Mediante relato dos profissionais de saúde, existem ações de educação em saúde direcionadas aos diabéticos e hipertensos, porém elas não abordam diretamente a prevenção de DRC e em muitas unidades as ações acontecem pontualmente no formato de grupos:

"Não, diretamente para doença renal não, mas sim para a questão do paciente diabético e hipertenso falando 
sobre as complicações da doença, aí sempre é falado a questão da alimentação, sobre a questão da ingesta hídrica, sobre a questão da necessidade da realização dos exames periódicos pra gente diagnosticar alguma alteração ainda na fase inicial, então mais ou menos voltado dessa forma, não diretamente pra doença renal, mas para hipertenso e diabético que acaba que a doença renal está ligada indiretamente" (P10)

A maioria destes profissionais, quando questionados a respeito da orientação sobre a prevenção de DRC voltada aos pacientes diabéticos e hipertensos durante atendimento ambulatorial, relataram não abordar o tema na prática clínica ou fazê-lo apenas quando se verifica alguma possível alteração renal instalada:

"Não, geralmente as nossas medidas é a questão da alimentação, uso da medicação correta, mas focada realmente pra doença renal não" (P05)

"Não, só quando a vê assim alguma coisa mais alterada né, a gente já começa a falar, mas já direcionar não, acho que é um erro né, já devia..." (P09)

Por outro lado, verificou-se que mais da metade dos profissionais nunca realizou capacitação para manejo da DRC na Atenção Primária ofertada pelo município, até pelo fato de não existir uma linha de cuidado implantada com esse fim e ao serem indagados sobre a necessidade de cursos e/ou capacitações voltada a essa temática, os relatos indicam a importância e indispensabilidade:

\begin{abstract}
"Sim, com certeza, acho muito essencial, tanto pra os enfermeiros quanto para os médicos, toda a equipe, porque são pacientes bem complicados e que quando eles vêm a ter complicações renais, já é num estágio bem avançado" (P11)
\end{abstract}

O papel da Atenção Primária na visão dos profissionais de saúde e gestores aponta a importância deste nível de atenção na promoção da saúde e na prevenção de doenças crônicas, bem como na adequada articulação e ordenação do cuidado, embora na prática existam dificuldades no cumprimento destas atribuições da APS:

"Um foco da atenção primária é a prevenção e a promoção de saúde, até a gente conseguir isso, conseguir esse objetivo, ainda tem muita caminhada, porque até hoje...ainda hoje nós estamos voltados à saúde como um todo, está voltada para aquela saúde curativa, então só procurar o médico, só procurar outro profissional de saúde, enfermeiro...nutricionista...quando estão acontecendo os efeitos do que a gente poderia ter prevenido né, então o foco da atenção primária seria prevenir, então a gente precisa de mais ações preventivas" (P04)

\section{DISCUSSÃO}

Os resultados deste estudo demons- 
traram fragilidades na assistência aos pacientes diabéticos e hipertensos, como a descontinuidade do cuidado envolvendo a atenção primária e especializada, no que diz respeito ao acesso aos exames e consultas especializadas, para a estratificação de risco destes pacientes. Alentidão para agendar consultas com médicos especialistas e agendar exames ou obter seus resultados, bem como o desabastecimento de fármacos e insumos para diabéticos foram considerados um entrave à capacidade de resolutividade da RAS em UBS no Distrito Federal, semelhante ao encontrado em nossa investigação ${ }^{14}$.

Dentro desse contexto de carência de especialistas, a inexistência de nefrologista foi citada pelos profissionais deste estudo e denota a realidade observada não apenas em nosso país, mas também em contextos internacionais, no qual se verifica uma maior densidade de nefrologistas em países de alta renda (> 15 por milhão de habitantes) quando comparado aos países de baixa renda ( $<5$ por milhão de habitantes). Uma baixa densidade de nefrologistas é preocupante, uma vez que são essenciais para liderar equipes multidisciplinares na prevenção e tratamento de doenças renais. A falta desses profissionais pode resultar em consequências adversas para as práticas e o desenvolvimento de políticas públicas voltadas principalmente aos grupos de risco com patologias renais ${ }^{15,16}$.

$\mathrm{O}$ vínculo fragilizado de diabéticos e hipertensos nas unidades investigadas foi notório, sendo restrita a busca por atendimento médico essencialmente para renovação de receitas de medicamentos, corroborando com os resultados encontrados em uma Unidade de APS na Paraíba, na qual $68 \%$ dos usuários procuravam o serviço somente para buscar a medicação de rotina, além disso, apenas 7,3\% dos usuários do Programa HiperDia realizaram 2 ou 3 atendimentos ao ano para consultas com os profissionais. Concomitantemente, cerca de 46,6 \% dos usuários acompanhados pelo mesmo programa na Bahia não compareciam a UBS há seis meses ou mais para o acompanhamento da(s) patologia(s) ${ }^{17,18}$.

A resistência ao tratamento e acompanhamento multiprofissional verificado entre os diabéticos e hipertensos na APS, eleva os riscos para complicações e/ou aparecimento de comorbidades renais. Vários estudos confirmam essa prática, revelando baixa adesão a dietas, à prática regular de atividade física e ao tratamento medicamentoso, que acomete principalmente os usuários com menor renda per capta e escolaridade ${ }^{19,20}$.

Comportamento semelhante foi observado entre os pacientes com DRC em TRS. Em pelo menos duas investigações, que avaliaram o manejo destes pacientes na APS, verificou-se que $49,7 \%$ destes não faziam o acompanhamento na sua UBS de referência, os profissionais desconheciam os portadores de DRC que fazem uso da hemodiálise em sua área de abrangência e somente tomam o conhecimento do caso quando ocorre a procura destes usuários na unidade ${ }^{21,22}$.

A carência de medicamentos e exames especializados apontados pelos pacientes com DRC, sendo necessário fazer uso de recursos próprios, configura-se como uma barreira para a vinculação destes com os serviços da APS. Uma investigação realizada em 125 países demonstrou que medicamentos para esses pacientes foram financiados gratuitamente em menos de $40 \%$ destes, com lacunas no tratamento particularmente acentuadas nos países de baixa e média renda. 
Este resultado foi confirmado por um estudo brasileiro com pacientes em TRS, o qual demonstrou que $41,7 \%$ tinham gastos atuais com medicamentos, demonstrando a debilidade do sistema público na garantia destes insumos e a importância do fortalecimento da rede de atenção perante a acessibilidade ao tratamento e o estado de saúde do paciente dialítico ${ }^{22,23}$.

Em uma visão global, quanto à capacidade e os recursos para o tratamento renal, muitos países ainda não têm acesso a diagnósticos básicos, a uma força de trabalho treinada em nefrologia, acesso universal à APS e às tecnologias de TRS. Isso se torna mais crítico mediante a forte possibilidade da doença renal contribuir para mais mortes do que as quatro principais doenças crônicas não transmissíveis visadas pelo atual "Plano de Ações Estratégicas para o Enfrentamento das Doenças Crônicas Não Transmissíveis (DCNT) no Brasil, 2011-2022", revelando a necessidade de sistemas de vigilância funcionais, uma vez que a importância global das doenças renais ainda não foi amplamente reconhecida, tornando-se uma doença negligenciada na agenda política global ${ }^{15}$.

Quanto à ausência de comunicação entre atenção primária e especializada relacionada à contrarreferência, um estudo abrangendo profissionais da APS e atenção especializada também demonstrou falhas ligadas à insuficiência de comunicação e ausência de retornos entre os trabalhadores dos serviços, o desconhecimento sobre os serviços da rede, a carência de educação permanente e a ausência de responsabilização dos profissionais envolvidos no cuidado. O sistema de referência e contrarreferência é um dos pontos estratégicos para consolidação do SUS, a fim de garantir a integralidade, uma vez que a fragilidade de comunicação entre os níveis de atenção provoca uma fragmentação da assistência, com consequente diminuição na resolução dos problemas de saúde da população assistida ${ }^{24,25}$.

Tem-se demonstrado a importância de implantação de medidas com ações de promoção da saúde e acompanhamento periódico de hipertensos e diabéticos a fim de reduzir os índices de falência renal. Um estudo verificou que $24,6 \%$ dos diabéticos e hipertensos avaliados em UBS encontravam-se com taxa de filtração inferior a $60 \mathrm{ml} / \mathrm{min}$, existindo, portanto, grande risco de já estarem com DRC em estágio inicial e evidenciando o encaminhamento secundário tardio relacionado à falta de conhecimento da epidemiologia da patologia, dos critérios diagnósticos e dos cuidados nos estágios iniciais da doença ${ }^{26}$.

Uma investigação feita com enfermeiros atuantes em UBS no Paraná demonstrou que nenhum profissional relatou treinamento específico para assistência em hemodiálise, gerando insegurança nos atendimentos e baixa interação perante qualquer intercorrência apresentada por estes pacientes. Concomitantemente, profissionais de UBS no Mato Grosso apresentaram dificuldades em relação a prescrições de cuidados de enfermagem próprios para fístula arteriovenosa em pacientes que realizavam hemodiálise, podendo prejudicar a assistência a esses pacientes ${ }^{21,27}$.

Com isso, reforça-se a importância da educação permanente em saúde (EPS) no âmbito da APS como uma estratégia para qualificar tecnicamente a atuação dos trabalhadores, mediante seu caráter transformador da realidade dos serviços e das necessidades da população baseada na problematização das 
questões reais e cotidianas. Mesmo diante de sua importância, estudos demonstraram que a EPS ainda não se configurou como ferramenta no cotidiano das equipes no contexto primário, seja pela dificuldade de sua compreensão entre os trabalhadores, seja pela falta de mecanismos de gestão que impulsionem e direcionem esta prática. Ainda são comuns processos formativos restritos a um conjunto de conteúdos, em sua maioria, com enfoque biologicista e com ênfase na prescrição de procedimentos e protocolos, resultando na consolidação de um modelo de atenção à saúde fragmentado e descontextualizado da realidade local. Portanto, torna-se imprescindível planejar e executar ações de incentivo à qualificação profissional, em serviço e continuamente, para aquisição de competências e habilidades necessárias ao dinamismo do cuidado na $\mathrm{AB}^{28,29}$.

Um estudo envolvendo profissionais brasileiros e canadenses demonstrou que os processos de trabalho de ambos os países pesquisados tem o seu foco baseado na produtividade do cuidado, com predominância de ações individuais e curativas por meio das consultas ambulatoriais, cobradas pelos gestores. A realização das práticas promotoras de saúde é mínima e desestimuladas ${ }^{30}$. É possível que este seja um motivo pelo qual se verificou a escassa existência de ações promotoras de saúde nas unidades estudadas no Município de Tauá, embora esse tópico não tenha sido abordado nas entrevistas, restringindo a compreensão desse aspecto. $O$ fato de o estudo ter sido realizado com uma amostra local torna as análises limitadas por representarem percepções de uma realidade em particular, não sendo possível generalizar os resultados.

\section{CONCLUSÃO}

O estudo revelou falhas na organização da assistência aos pacientes diabéticos e hipertensos na APS e a sua relação com a atenção especializada, sobressaindo-se o acesso insuficiente aos medicamentos e exames especializados, especialmente em nefrologia; bem como o vínculo fragilizado e a baixa adesão dos pacientes ao tratamento multiprofissional no componente primário e ausência de contrarreferência.

A investigação trouxe à tona lacunas na abordagem preventiva da atenção básica, evidenciando ausência de ações direcionadas à prevenção de DRC nos principais grupos de risco tanto nos atendimentos ambulatoriais quanto nas ações coletivas, apesar dos profissionais e gestores reconhecerem a importância desta atribuição da atenção básica. Por outro lado, os profissionais citam a necessidade de educação permanente sobre o manejo da DRC no âmbito da APS.

Diante dos resultados expostos, aponta-se a imprescindibilidade de fortalecimento da RAS no município estudado, fortalecendo as ações de EPS e a construção conjunta de planos de cuidado para os pacientes diabéticos e hipertensos envolvendo os níveis de atenção do SUS, fortalecendo o papel da APS como ordenadora e coordenadora do cuidado. Para isso, faz-se necessária a modelagem e implantação de uma linha de cuidado voltada à prevenção, diagnóstico e tratamento das doenças renais crônicas com o intuito de controlar efetivamente o avanço dessas condições crônicas com alto grau de limitações e alto custo para os serviços de saúde públicos. 


\section{REFERÊNCIAS}

1. Wong LY, Liew AST, Weng WT, Lim K, Vathsala A, To MPHS. Projecting the Burden of Chronic Kidney Disease in a Developed Country and Its Implications on Public Health. International Journal of Nephrology. 2018; 5196285.

2. Hill N, Fatoba ST, Oke JL, Hirst JA, O'callaghan CA, Lasserson DS, Richard Hobbs FD. Global Prevalence of Chronic Kidney Disease - A Systematic Review and Meta-Analysis. PLOS ONE. 2016; 11(7).

3. BRASIL. Ministério da Saúde. Secretaria de Atenção à Saúde. Departamento de Atenção Especializada e Temática. Diretrizes Clínicas para o Cuidado ao paciente com Doença Renal Crônica - DRC no Sistema Único de Saúde. Secretaria de Atenção à Saúde. Departamento de Atenção Especializada e Temática. - Brasília: Ministério da Saúde, p.:37, 2014.

4. Thomé FS, Sesso RC, Lopes AA, Lugon JR, Martins CT Inquérito Brasileiro de Diálise Crônica 2017. J. Bras. Nefrol. Inquérito Brasileiro de

5. Amorim RG, Guedes GS, Vasconcelos SML, Santos JCF Doença Renal do Diabetes: Cross-Linking entre Hiperglicemia, Desequilíbrio Redox e Inflamação. Arq Bras Cardiol. 2019; 112(5):577-587.

6. Coelho SR, Castro LG, Miamae LBA, Ferreira TS, Pinheiro MA, Coelho MR. A influência da HAS na fisiopatogenia da DRC: uma revisão sistemática. Rev de Patologia do Tocantins. 2019; 6(2):57-60

7. Oliveira APB, Schmidt DB, Amatneeks TM, Santos JC, Cavallet LHR, Michel RB. Qualidade de vida de pacientes em hemodiálise e sua relação com mortalidade, hospitalizações e má adesão ao tratamento. J. Bras. Nefrol. 2016; 38(4):411420.

8. Duarte L, Hartmann SP. A autonomia do paciente com doença renal crônica: percepções do paciente e da equipe de saúde. Rev. SBPH. Rio de Janeiro. 2018; 21(1).

9. Alcalde PR, Kirsztajn GM. Gastos do Sistema Único de Saúde brasileiro com doença renal crônica. J.Bras.Nefrol. 2018; 40(2):122-129.

10. Marinho AWGB, Penha AP, Silva MT, Galvão TF. Prevalência de doença renal crônica em adultos no Brasil: revisão sistemática da literatura. Cad. Saúde Colet. 2017; 25(3): 379-388.

11. BRASIL. Portaria $n^{0} 1.675$, de 7 de junho de 2018. Altera a Portaria de Consolidação $n^{\circ}$ 3/GM/MS, de 28 de setembro de 2017, e a Portaria de Consolidação n 6/GM/MS, de 28 de setembro de 2017, para dispor sobre os critérios para a organização, funcionamento e financiamento do cuidado da pessoa com Doença Renal Crônica - DRC no âmbito do Sistema Único de Saúde - SUS. Diário Oficial da União, Brasília, DF, 07 de junho de 2018, Seção I, p. 148-151.

12. Strauss A, Corbin J. Pesquisa Qualitativa: técnicas e procedimentos para o desenvolvimento de teoria fundamentada. $2^{\mathrm{a}}$ ed., Porto Alegre, Artmed, 2008.

13. Minayo MCS (org.). Pesquisa social: teoria, método e criatividade. 29. ed. Petrópolis, RJ: Vozes, 2010.

14. Carmo KS, Medeiros M, Almeida OAE, Rehem TCMSB, Zanchetta MS, Santos WS. Rede de atenção à saúde na perspectiva de usuários com diabetes. Cien Cuid Saúde J. 2019; 18(3).

15. Crews DC, Bello AK, Saadi G. Editorial do Dia Mundial do Rim 2019 - impacto, acesso e disparidades na doença renal. J Brasileiro de Nefrologia. 2019; 41(1):1-9.

16. Qarni B, Osman MA, Levin A, Feehally J, Harris D, Jindal K et al. Kidney care in low- and middle-income countries. Clin Nephrol. 2020; 93(1):21-30.
17. Negreiros RV, Camêlo ES, Sabino TC, Santos MS, Aguiar DC Importância do programa hiperdia na adesão ao tratamento medicamentoso e dietético em uma Unidade de Saúde da Família (USF). Revista da Universidade do Vale do Rio Doce. 2016; 14(2):403-411.

18. Araújo MCF, Alves MS, Padre PMM, Andrade DM, Silva ACM Barretto LP et al. Perfil de Não Adesão ao Tratamento de Usuários com Diabetes e Hipertensão em uma Unidade de Saúde da Família. Ensaios e Ciência: Ciências Biológicas, Agrárias e da Saúde, 2016. 20(1):43-48.

19. Gewehr DM, Bandeira VAC, Gelatti GT, Colet CF, Oliveira KR. Adesão ao tratamento farmacológico da hipertensão arterial na Atenção Primária à Saúde. Saúde debate 2018 42(116):179-190.

20. Moreira SFC, Donato KS, Silva LED, Lima KLL, Pelazza $\mathrm{BB}$, Borges $\mathrm{CJ}$ et al. Avaliação dos fatores relacionados à adesão de pacientes com diabetes mellitus ao tratamento. Rev. Itinerarius Reflectionis. 2018; 14(4):01-19.

21. Castoldi ARDS, Garcia SM, Hartwig SV. Assistência de enfermagem a pacientes em hemodiálise na atenção básica. Rev Eletronica Gestão \& Saúde. 2016; 7(3):1200-1215.

22. Spigolon DN, Teston EF, Costa MAR, Maran E, Souza RR Moreira Neto A. Acessibilidade ao tratamento e estado de saúde de pacientes hemodialíticos. Rev de Enfermagem UFPE. 2018; 12(7):1853-1858.

23. Htay H, Alrukhaimi M, Ashuntantang GE, Bello AK, Bellorin-Font $E$ et al. Global access of patients with kidney disease to health technologies and medications: findings from the Global Kidney Health Atlas project. Kidney International. 2018; 8(2Supl):64-73

24. Brondani JE, Leal FZ, Potter C, Silva RM, Noal HC, Perrando MS. Desafios Da Referência E Contrarreferência Na Atenção Em Saúde Na Perspectiva Dos Trabalhadores. Cogitare Enfermagem. 2016; 21(1):1-8.

25. Silva KAB, Juliani CMCM, Spagnuolo RS, Mori NLR, Dias Baptista SCP, Martin LB. Desafios no processo de referenciamento de usuários nas redes de atenção à saúde: perspectiva multiprofissional. Cienc. Cuid. Saúde. 2018; 17(3).

26. Dallacosta FM: Masson NHB, Pasquali DCS. Doença Renal: Detecção Precoce Em Grupos De Hiperdia. Saúde.com. 2018; 14(2):1197-1201

27. Fernandes AR, Hartwig, SV, Silva EC, Alencar BT. Identificação da fístula arteriovenosa e suas complicações pelos enfermeiros dos serviços de entrada de Cáceres-MT. Rev de APS. 2019; 21(3):408-417.

28. Bispo Júnior JP Moreira DC Educação permanente e apoio matricial: Formação, vivências e práticas dos profissionais dos Núcleos de Apoio à Saúde da Família e das equipes apoiadas. Cad Saude Publica. 2017; 33(9).

29. Moreira KS, Lima CA, Vieira MA, Costa SM. Educação Permanente e Qualificação Profissional Para Atenção Básica. Saúde e Pesquisa. 2017; 10(1):101-109.

30. Heidemann ITSB, Cypriano CC, Gastaldo D, Jackson S, Rocha CG, Fagundes E. Estudo comparativo de práticas de promoção da saúde na atenção primária em Florianópolis, Santa Catarina, Brasil e Toronto, Ontário, Canadá. Cad Saude Publica. 2018; 34(4).

CORRESPONDÊNCIA

Elayne Cristina Matias Nóbrega

Endereço postal: Rua François Loiola,

n.7, Tauazinho,

Tauá, Ceará. CEP 63660-000

E-mail: elaynenobrega.nutri@gmail.com 